\title{
La indecencia del trabajo informal en Colombia*
}

\section{The indecency of informal work in Colombia}

\begin{abstract}
Omar Ernesto Castro Guiza'
1 Profesor Investigador Universidad Cooperativa de Colombia-Ibagué. Correo electrónico: omarc75@hotmail.com

\section{JUSTICIA}

ARTíCULO DE

INVESTIGACIÓN

Recibido: $15-07-17$

Aceptado: $30-08-17$

Publicado: $18-01-18$

DOI:

\section{RESUMEN}

Este estudio analiza el concepto de informalidad por subsistencia, descrito en la Ley 1429 de 2010 de Colombia, causas generadoras del fenómeno, lineamientos de los organismos internacionales y nacionales. Además, examina las estrategias jurídicas implementadas con el objetivo de reducir las altas tasas de informalidad en el país, pretendiendo ofrecer garantías laborales mínimas a los trabajadores mediante el fomento y creación de empleos formales. Igualmente, se revisan las propuestas internacionales recomendadas por la OIT desde el Programa Mundial de Trabajo Decente, para mitigar el fenómeno de la informalidad laboral. Utilizando un método de investigación explicativo, se pretende plantear la necesidad de enrutar el trabajo informal hacia el trabajo decente.
\end{abstract}

https://doi.org/10.17081/just.23.33.2889
Palabras clave: Formalización laboral, informalidad, trabajo decente y subsistencia.

\section{ABSTRACT}

This study analyzes the concept of informality by subsistence, described in Law 1429 of 2010 of Colombia, causes that generate the phenomenon, guidelines of international and national organizations. In addition, it examines the legal strategies implemented with the objective of reducing the high rates of informality in the country, seeking to offer minimum labor guarantees to workers through the promotion and creation of formal jobs. Likewise, the international proposals recommended by the OIT from the Global Decent Work Program are reviewed to mitigate the phenomenon of labor informality. Using an explanatory research method, it is intended to raise the need to route informal work towards decent work.

Key words: Formalization of labor, informality, decent work and subsistence.

Artículo de investigación como avance del proyecto de tesis doctoral "El camino hacia el trabajo decente para los informales en Colombia", que desarrolla el autor en la Universidad Santo Tomás de Bogotá.

Cómo citar este artículo:

Castro Guiza, O. E. (2018). La indecencia del trabajo informal en Colombia. Revista Justicia, 23(33), 200-223.

https://doi.org/10.17081/just.23.33.2889 


\section{INTRODUCCIÓN}

El trabajo concebido como un derecho humano, ha sido incluido dentro de la categoría de los derechos económicos, sociales y culturales, así como lo describe el pacto internacional vigente, el cual lo señala en el artículo 7 en los siguientes términos: "Los Estados Partes en el presente Pacto reconocen el derecho de toda persona al goce de condiciones de trabajo equitativas y satisfactorias". Asimismo, la citada norma expresa que el trabajo debe garantizar una remuneración adecuada a los trabajadores, condiciones de existencia digna para ellos y sus familias, igualdad de género en el ámbito laboral, seguridad e higiene en el trabajo y tiempo para el descanso y la recreación (Organización de Naciones Unidas, 1966).

No obstante, a partir del fenómeno de Globalización de la economía iniciado en la década de los años sesenta, el mundo del trabajo ha sido afectado significativamente, observándose un aumento de la flexibilidad en las modalidades de ejecución de actividades laborales, desempleo y precarización del trabajo. Es así, como han surgido diversas formas de ejercer trabajo, tales como, el empleo a tiempo parcial, la subcontratación y el trabajo asociado, entre otras, que precarizan los derechos de los trabajadores. Sin embargo, el aumento acelerado del trabajo informal en el mundo en las últimas cuatro décadas, lo ha convertido en la modalidad de trabajo más común, por ofrecer la solución inmediata para la generación de ingresos y la subsistencia de los individuos. Sin embargo, el trabajo en el sector informal, así como lo comentó en un primer concepto la Organización Internacional del Trabajo-OIT, no ofrece condiciones laborales dignas, ni justas para el trabajador, y por el contrario su escenario de trabajo se precariza; observándose falta de protección social, remuneración baja y desigualdad frente a los trabajadores formales (Organización Internacional del Trabajo, 1991).

Con todo, la presente investigación centra su objeto de estudio en el concepto legal de informalidad por subsistencia, definido en Colombia por la Ley 1429 de 2010. Así, como se explicará más adelante, Colombia en los últimos años ha tenido un comportamiento muy similar a la generalidad de los países de América Latina y el Caribe, sobre la problemática concebida dese el alto índice de trabajo informal en el país, especialmente para aquellas personas que en situación de pobreza, tratan de satisfacer las necesidades primarias de vida, desde el desempeño de actividades informales. Con base en lo anterior, se pretende analizar si el Estado colombiano ha logrado avances significativos en el camino hacia garantizar trabajo decente para los trabajadores enmarcados dentro del concepto de informalidad por subsistencia. Entonces, la pregunta principal de la investigación es: 
¿Cuál es el contenido y ámbito de aplicación del concepto de informalidad por subsistencia del empleo, descrito en el numeral 3 del literal a, del artículo 2 de la Ley 1429 de 2010, en concordancia con el concepto de trabajo decente definido por la OIT?

En la presente investigación se plantea el uso de un método de investigación cualitativo desde la Hermenéutica, teniendo en cuenta que a través de este método, se busca comprender los textos desde su ejercicio intencional y conceptual; se trata entonces, de desarrollar la inteligibilidad del discurso, contenida en un texto, para traspasar las fronteras de la palabra y encontrarle el sentido a las mismas (Cárcamo, 2005).

Asimismo, el enfoque del estudio será descriptivo y explicativo, teniendo en cuenta el concepto de Hernández, Fernández y Baptista (2003), los estudios descriptivos se centran en recolectar datos que muestran un evento, una comunidad, un fenómeno, hecho, contexto o situación. Asimismo, los autores afirman que los estudios explicativos van más allá de la descripción de conceptos o fenómenos o del establecimiento de relaciones entre conceptos; están dirigidos a responder a las causas de los eventos físicos o sociales.

\section{DISCUSIÓN}

La OIT ha entendido el contexto en que se desempeñan los trabajadores informales bajo el concepto de "economía informal," el cual es definido como:

Las muy pequeñas unidades de producción y distribución de bienes y servicios, situadas en las zonas urbanas de los países en desarrollo; dichas unidades pertenecen casi siempre a productores y trabajadores independientes que a veces emplean a miembros de la familia o a algunos asalariados o aprendices. Estas unidades disponen de muy poco o ningún capital; utilizan técnicas rudimentarias y mano de obra escasamente calificada; por lo que su productividad es reducida; quienes trabajan en ellas suelen obtener ingresos muy bajos e irregulares y su empleo muy inestable. (Organización Internacional del Trabajo, 1991, p. 4)

Lo que da lugar a pensar, que los elementos distintivos de la economía informal son, por una parte, la estructura incipiente de la organización productiva y, por otra, la inestabilidad y escasez de garantías en el empleo. Es por ello que la OIT, reconoció la evolución del concepto de trabajo informal y desde hace varios años ha utilizado la expresión "sector informal de la economía" para describir las diversas actividades de trabajadores pobres que no son contem- 
pladas, protegidas, ni reguladas por las autoridades públicas, tales como las ventas callejeras, trabajo doméstico, trabajos de empresas explotadoras, trabajos a domicilio, trabajos independientes, microempresas, entre otros.

Asimismo, la OIT distingue dos principales características de los trabajadores informales: la primera se refiere a que este tipo de trabajadores no están reconocidos, ni protegidos dentro de los marcos jurídicos y reglamentarios de los Estados y en segundo lugar, están afectados por un alto nivel de vulnerabilidad, siendo sus labores muy inestables y sus ingresos muy bajos e irregulares (Oficina Internacional del Trabajo, 2002).

Para reforzar la explicación, se hace necesario mencionar las dos principales teorías sobre la informalidad laboral. La primera es la teoría estructuralista que afirma que es el resultado de un escaso desarrollo del sector moderno de la economía, de tal manera que este no alcanza a absorber toda la fuerza laboral disponible, la población excedente, sea educada o no, se ve forzada a laborar en actividades informales de baja remuneración o cae en el desempleo. La segunda teoría, la institucionalista centra su explicación en los altos costos que el Estado y en general las instituciones le imponen al funcionamiento, legalización y desempeño de las empresas, lo cual impide su desarrollo y generación de empleos formales (Uribe, \& otros, 2006).

Ahora bien, para sustentar la realidad de la problemática generada por el aumento desmesurado del trabajo informal en el mundo, basta revisar las cifras socializadas por la OIT. En primer lugar, se menciona que el continente con más alto índice de trabajo informal en el mundo es el africano, el cual alcanzó en los últimos años casi el $80 \%$ del empleo no agrícola, más del $60 \%$ del empleo urbano y más de los $90 \%$ de los nuevos puestos de trabajo (Charmes, 2000). En Asia, la proporción de trabajadores informales del sector no agrícola iba del 45 al $85 \%$, y la del empleo informal urbano del 40 al $60 \%$. Por su parte, la Unión Europea registra el índice más bajo de trabajo informal en el mundo, oscilando entre el $7 \%$ al $19 \%$ del empleo declarado (Oficina Internacional del Trabajo, 2002).

\begin{tabular}{ccc}
\multicolumn{3}{c}{ Índice de informalidad laboral por continente } \\
\hline Continente & Sector urbano & Sector agrícola \\
\hline África & $60 \%$ & $80 \%$ \\
\hline Asia & $40-60 \%$ & $45-85 \%$ \\
\hline Europa & & $19 \%$ aprox. \\
\hline América Latina & $47,7 \%$ & $50 \%$ \\
\hline
\end{tabular}

Fuente: OIT, Conferencia 90, 2002, El trabajo decente y la economía informal 
El caso de América Latina y el Caribe exige una mayor profundidad en el estudio, en primer lugar, porque es la región geográfica en donde se ubica el país objeto de estudio de esta investigación, Colombia, y en segunda medida, porque el trabajo informal genera la principal problemática en asuntos laborales de la región. En la zona, se registra para el año 2012, un índice de informalidad laboral no agrícola del $47,7 \%$, lo que indica que aproximadamente 130 millones de personas ocupadas ejercen empleos informales. Las actividades que representan las cifras presentadas se distribuyen así: un $31 \%$, corresponde a empleo en el sector informal, un $11,6 \%$, es empleo informal en el sector formal, y un 5,1\%, está constituido por empleo informal en actividades de servicios domésticos.

\begin{tabular}{cc} 
& Índice de informalidad laboral \\
\hline \multirow{2}{*}{$\begin{array}{c}\text { América Latina y el Caribe } \\
\text { año } 201247,7 \%\end{array}$} & $31 \%$ Trabajadores en el sector informal \\
\cline { 2 - 2 } & $11,6 \%$ Empleo informal en el sector formal \\
\hline
\end{tabular}

Fuente: OIT-ALC, Notas sobre formalización, 2014

Asimismo, en la región se identifican como trabajadores informales el $84 \%$ de los trabajadores por cuenta propia, el $79 \%$ de los trabajadores domésticos, el $33 \%$ de los trabajadores del sector privado y el $60 \%$ de las personas que laboran en pequeñas empresas. Las trabajadores con menor nivel de educación, los más jóvenes, las mujeres y los más pobres, son las personas con mayor ejercicio de trabajos informales (Oficina Regional para América Latina y el Caribe, 2014).

\begin{tabular}{cc}
\hline & Índice de trabajadores informales por sectores \\
\hline \multirow{3}{*}{$\begin{array}{c}\text { América Latina y el Caribe } \\
\text { año } 2012\end{array}$} & $84 \%$ Trabajadores por cuenta propia \\
\cline { 2 - 2 } & $79 \%$ Trabajadores del servicio doméstico \\
\cline { 2 - 2 } & $33 \%$ Trabajadores del sector privado \\
\hline
\end{tabular}

Fuente: OIT-ALC, Notas sobre formalización, 2014

Sumado a lo anterior, se distinguen los países de la región latinoamericana y del Caribe con las tasas más altas de informalidad: en primer lugar está Honduras 70,7 \%, luego Perú con 68,8 \%, Paraguay con 65,8 \%, El Salvador con $65,7 \%$, Colombia con 56,8 \%, México con 54,2 \%, Ecuador con 52,2 \% y República Dominicana con 50 \% (ibídem).

Ahora bien, al analizar la problemática de la informalidad en Colombia, se colige que ha seguido la misma tendencia de la región latinoamericana, se ha determinado que en el país, el fenómeno de la informalidad laboral ha 
aumentado en las últimas dos décadas, registrando un índice igual o mayor al $50 \%$, frente a la totalidad de las actividades que se consideran empleo. Al respecto, se han observado las consecuencias negativas para los trabajadores informales referentes a las condiciones en que desempeñan sus labores y su falta de protección, validando la tendencia regional y mundial descrita por OIT en su Conferencia 78, sobre la precarización del empleo en el sector informal.

Actualmente, y según el informe del Departamento Administrativo Nacional de Estadística (DANE), para el trimestre móvil octubre-diciembre de 2016, del total de ocupados, el 48,7\%, se clasificó como informal. Según la rama de actividad el $42,2 \%$ se consolidó en el comercio, hoteles y restaurantes; también se encuentra que según la posición ocupacional el trabajador por cuenta propia acumuló el $60,1 \%$ de la población, en cuanto al nivel educativo encontramos que el $51,7 \%$ de los trabajadores informales tenía estudios secundarios y el principal lugar de informalidad es el local fijo con un $41,3 \%$; en cuanto a la seguridad social el mismo informe nos indica que el 93,4 \% de los ocupados tuvieron acceso a algún régimen de seguridad social en salud, asimismo que del número total de ocupados $(66,0 \%)$ estaba afiliado al régimen contributivo, el $22,7 \%$ al régimen subsidiado y el 2,6 \% al régimen especial; finalmente el informe ejecutivo nos señala que el $48,2 \%$ de los encuestados estaban cotizando pensión en el periodo referenciado (Departamento Administrativo Nacional de Estadística, 2017).

Sobre el tema, la Procuraduría General de la Nación realizó un estudio, el cual llamó "Trabajo Digno y Decente en Colombia," allí, afirma que el contexto laboral colombiano es preocupante ya que de cada diez trabajadores seis no tienen un empleo digno, y que además el $63 \%$ de los trabajadores del país ejercen labores de baja calidad, lo que se representa en desigualdad y desprotección laboral, evidenciando que nuestro país es uno de los que tiene mayor disparidad en América Latina. Igualmente, se observa que el $46 \%$ de los ingresos de los trabajadores son menores de un salario mínimo mensual y que la forma de vinculación por intermediación ha subido en los últimos años (Procuraduría General de la Nación, 2012).

Continuando con el caso colombiano, la informalidad se ha convertido en una salida rápida a la gran problemática del desempleo en Colombia y América Latina. Indica, además, que el desempleo en las últimas décadas ha crecido debido a diversos fenómenos a saber: la violencia que ha obligado a las personas del sector rural a desplazarse a las ciudades, el proceso de recesión económica 
de las décadas de los 80 y 90, los altos costos de formalización para pequeñas empresas, las altas cargas tributarias, el decrecimiento de la mortalidad infantil y la fecundidad, la discriminación laboral hacia las mujeres, la subutilización de la fuerza de trabajo, la exigencia de mano de obra calificada para trabajos que no lo requieren, el desempeño de cargos por parte de personas sin la debida preparación técnica y la creación de empleos temporales e intermitentes con baja calidad y remuneración.

Con todo, y atendiendo las distintas problemáticas laborales globales, entre ellas la del trabajo informal, la OIT diseñó el Programa Mundial de Trabajo Decente (en adelante PTD), presentado en la 87 reunión de junio de 1999 en Ginebra-Suiza por la Conferencia Internacional del Trabajo. La noción de trabajo decente se expuso por primera vez, en 1999 por el entonces director general de la OIT, el chileno Juan Somavia, en los siguientes términos: "Trabajo productivo en condiciones de libertad, equidad, seguridad y dignidad, en el cual los derechos son protegidos y que cuenta con remuneración adecuada y protección social" (Somavia, 1999, p. 4).

Para Ghai (2003), el PTD "abarca todas las clases de trabajo y tiene facetas cualitativas y cuantitativas. La idea de trabajo decente es válida tanto para los trabajadores de la economía regular como para los trabajadores asalariados de la economía informal, los trabajadores autónomos (independientes), y los que trabajan a domicilio" (p. 1).

En el Programa de Trabajo Decente convergen cuatro objetivos principales: el empleo, los derechos fundamentales en el trabajo, la protección social y el diálogo social, los cuales son los referentes orientadores en el quehacer internacional de la OIT. Respecto al primer objetivo, el empleo, en la reunión Conferencia 87, la OIT afirma que los Estados deben promover posibilidades de trabajo que permitan el desarrollo personal del individuo y que generen la oportunidad de ganarse la vida decorosamente. Además, que se deben implementar políticas que disminuyan el desempleo y el subempleo, y promocionen los derechos en el trabajo.

Para Ghai (2003):

El concepto "abarca todas las clases de trabajo y tiene facetas cualitativas y cuantitativas. La idea de trabajo decente es válida tanto para los trabajadores de la economía regular como para los trabajadores asalariados de la economía informal, los trabajadores autónomos (independientes), y los que trabajan a domicilio.' (p. 1) 
En lo concerniente a los derechos fundamentales en el trabajo, la Conferencia hace alusión a su protección y garantía para los trabajadores, tanto en el sector formal como en el sector informal (no estructurado), sin distinción del lugar o actividad lícita que se desempeñe. En relación al tercer objetivo del PTD, la protección social, el mismo documento plantea que este debe atender las causas y mitigar las consecuencias que generan vulnerabilidad y retiro de las personas de sus trabajos, independientemente que se originen desde situaciones de desempleo, vejez, padecimientos de salud u otras.

Desde el diálogo social, se fomenta la participación y libre asociación, a través de organizaciones democráticas, así como la comunicación entre entes gubernamentales, empleadores y trabajadores, que admitan la conciliación de los distintos intereses de cada una de las partes, y permitan un Trabajo Decente para todos. El ideal, entonces del PTD es conminar a los Estados para que implementen políticas públicas dirigidas al aumento de los índices de empleos formales en condiciones dignas, garantes de los derechos de los trabajadores y con protección social para ellos y sus familias.

En América Latina, se han propuesto diversas estrategias políticas, económicas y jurídicas para enrutar a los Estados hacia el alcance de los objetivos del PTD. En relación a ello, se han identificado siete estrategias políticas principales para hacer transición del empleo informal al formal, las cuales se mencionan a continuación:

- Generación de empleo de calidad y estrategias de crecimiento

- Entorno normativo; incluida la observancia de las normas internacionales del trabajo y los derechos fundamentales

- Organización, representación y diálogo social

- Fomento de la igualdad y lucha contra la discriminación

- Medidas de apoyo a la iniciativa empresarial, competencias profesionales y financiación

- Ampliación de la protección social: establecimiento de pisos de protección social y sistemas de seguridad social

- Estrategias de desarrollo local

Según la OIT, en la región latinoamericana, algunos países han implementado mecanismos globales desde las políticas mencionadas et supra, y en alguna medida, han logrado detener el acelerado aumento de la informalidad; y empiezan lentamente el tránsito hacia la formalidad laboral, sin embargo, no es suficiente, pues aún son muy altos los índices de trabajo informal en la 
región y se sigue notando una correlación directa entre informalidad y pobreza (Oficina Regional para América Latina y el Caribe, 2013, p. 65).

Para analizar el caso colombiano referente al tema, hay que mencionar que el país es uno de los ciento ochenta y tres Estados miembros activos de la OIT, además, ostenta la calidad de miembro fundador desde su creación en 1919. Atendiendo tal vinculación, el país está obligado a observar las exigencias impuestas por el organismo internacional desde su constitución y las normas generadas desde allí, con el ánimo de lograr los objetivos que se ha trazado desde sus orígenes, dirigidos principalmente a la búsqueda de la justicia social y la paz universal, a través de la promoción de los derechos laborales, el fomento del trabajo decente, el avance en las condiciones de seguridad social de los ciudadanos y el diálogo permanente en los escenarios laborales de los pueblos (Organización Internacional del Trabajo, 2012).

Es pertinente indicar que Colombia ha ratificado 61 convenios de los 189 adoptados por OIT, todos por la vía de ley, destacándose que los 8 convenios fundamentales se encuentran allí; y que el último data de 2014 cuando se ratificó el Convenio número 189, sobre las trabajadoras y los trabajadores del servicio doméstico (Organización Internacional del Trabajo, 2014).

Ahora bien, para determinar si Colombia ha atendido con suficiencia las obligaciones contraídas frente a OIT, es necesario revisar los informes más recientes del propio organismo, por medio de los cuales se hace el seguimiento y evaluación a las estrategias y normas informadas por los propios Estados, con el ánimo de cumplir con sus compromisos en materia de Trabajo Decente. Al respecto se enuncia que la OIT, hace control de las obligaciones mencionadas, a través de la Comisión de aplicación de normas (órgano de control); desde la enunciada dependencia el Estado colombiano ha sido citado 18 veces, para dar cuenta de su reiterada omisión a la aplicación de los convenios, especialmente del 87 y 98, que versan sobre libertad sindical, derecho de negociación colectiva y derecho de sindicación (Ministerio de Relaciones Exteriores y Ministerio de la Protección Social, 2012).

Como consecuencia de lo anterior, Colombia, adoptó medidas en los últimos años, tendientes a disminuir la violación de los derechos referenciados y a mejorar el diálogo social entre los actores de las relaciones laborales, acompañándose de OIT para ello. En virtud de tal necesidad, se creó el programa de Especial Cooperación para Colombia, el cual se desarrolló entre 2002 y 2005, y permitió que se disminuyera la violencia hacia los sindica- 
listas y se aumentara la protección a sus derechos al interior del país, según informe presentado a OIT en 2009. Este mismo documento facilitó que la propia comisión declarara a Colombia como País en progreso, frente a los compromisos en materia laboral. Con el objetivo de seguir avanzando en el proceso para la mejora de la situación laboral, el país se acompañó del Estado canadiense a través del programa PLADES (Programa Laboral de Desarrollo), el cual involucra además de Colombia, a Perú, Ecuador y Bolivia. El propósito del programa está dirigido a mejorar el déficit de Trabajo Decente en la región Andina.

Teniendo en cuenta el proyecto referenciado, se realizó un diagnóstico sobre el tema laboral en la región. Los datos observados allí, reflejan que en la zona estudiada, el país con más desempleo es Colombia con un índice de 11,6\% para el año 2008, seguido por Perú con un 8,6 \%. Sin embargo, la situación que más afecta a la región no es la tasa de desempleo, sino de subempleo y empleo informal, que dan cuenta de más de la mitad de los ocupados. Estos trabajadores están caracterizados por un alto grado de vulnerabilidad, debido a la falta de regulación normativa respecto a las actividades por ellos ejercidas, así como al bajo índice de protección social. Sobre este último aspecto, el informe data que en Colombia para 2009 de cada 100 trabajadores/as solo 30 son afiliados cotizantes a una entidad pensional, 31 al Sistema de Riesgos Profesionales, 41 como cotizantes en salud y 31 en Cajas de Compensación Familiar, evidenciándose que más de 12 millones de empleados están desprovistos de protección social (Villavicencio y otros, 2010).

El Estado colombiano ha expedido en los últimos años variadas normas laborales destinadas a mejorar el contexto en el trabajo para sus conciudadanos; sin embargo, dichas reglas no obedecen a la implementación de una política pública nacional del Programa de Trabajo Decente, sino a medidas inmediatistas que pretenden disminuir las situaciones más relevantes de afectación a los trabajadores, pero que en muchas ocasiones no tienen aplicabilidad. Obsérvese, cómo desde la Carta Política de 1991, el trabajo como derecho se constitucionalizó como fundamental según lo denota el artículo 25 y que lo anuncia como un derecho y un deber del ciudadano, pero con tutela para su ejercicio; asimismo se fijaron unos principios laborales mínimos y fundamentales enlistados en el artículo 53 ibídem.

Para el desarrollo de los enunciados constitucionales y el avance en pro de mejorar el contexto laboral en el país, el Estado ha expedido en los últimos años algunas normas laborales, tales como: La Ley 1010 de 2006 (sobre acoso laboral), Ley 1221 de 2008 (sobre teletrabajo), Ley 1429 de 2010 (sobre 
generación y formalización del empleo), Ley 1450 de 2011 (Plan Nacional de Desarrollo), Decreto 2025 de 2011 (sobre contratación a través de cooperativas y precooperativas de trabajo asociado), Ley 1438 de 2011 (reforma al sistema de salud), Ley 1468 de 2011 y Ley 1822 de 2017 (sobre licencia de maternidad), la Ley 1496 de 2011(sobre equidad salarial para la mujer), Ley 1788 de 2016 (prima de servicios para trabajadoras del servicio doméstico).

Ahora bien, para apaciguar la crisis del empleo y el aumento de la informalidad laboral en Colombia, se puso en vigencia la Ley 1429 de 2010, "Por la cual se expide la Ley de Formalización y Generación de Empleo." Desde ella, se pretende generar empleo de manera masiva, a través de incentivos tributarios y beneficios económicos a las empresas, que logren vincular a los trabajadores/as, con más tendencia a no ser contratados, esto es, los jóvenes, mujeres y personas mayores de 40 años. De la misma manera, se aspira con la implementación de la norma, que las pequeñas empresas ${ }^{2}$ que no están formalizadas, lo hagan, y así lograr que sus trabajadores/as gocen de todas y cada una de las garantías laborales y de protección social (Congreso de Colombia, 2010).

La mencionada norma ofrece el concepto legal de informalidad laboral en Colombia, definiéndolo en su artículo segundo, desde dos ópticas, así: a) Informalidad por subsistencia: Es aquella que se caracteriza por el ejercicio de una actividad por fuera de los parámetros legalmente constituidos, por un individuo, familia o núcleo social para poder garantizar su mínimo vital. b) Informalidad con capacidad de acumulación: Es una manifestación de trabajo informal que no necesariamente representa baja productividad. La informalidad por subsistencia. Debe entenderse el conjunto de actividades de trabajo cuyo fin principal es la supervivencia de una persona u hogar a través de la producción o venta directa de bienes y servicios, tales como ventas callejeras ambulantes, en local fijo, entre otras. Este tipo de trabajos son generalmente realizados por trabajadores por su cuenta propia de manera individualizada o en algunas ocasiones en grupos familiares, cuyos integrantes aportan su fuerza de trabajo, con el ánimo de generar ingresos, que generalmente son precarios e insuficientes para satisfacer sus necesidades básicas de vida.

En cuanto al concepto de informalidad con capacidad de acumulación, se podría entender, como lo manifiestan los autores anteriormente citados, desde una dualidad de definiciones; la primera concebida como informalidad

2 Ver artículo 2: "Para los efectos de esta ley, se entiende por pequeñas empresas aquellas cuyo personal no sea superior a 50 trabajadores y cuyos activos totales no superen los 5.000 salarios mínimos mensuales legales vigentes." 
de explotación dependiente, cuyo objetivo es lograr mayor flexibilidad en los procesos productivos y menores costos en las empresas del sector formal, por medio de contrataciones no registradas y de subcontrataciones de microemprendimientos informales; y la segunda como informalidad de crecimiento que se centra en lograr acumulación de capital en empresas pequeñas, gracias a la movilización de redes solidarias, mayor flexibilidad laboral y costos más bajos (ibídem).

Como ya se dijo et supra, los elementos distintivos de la economía informal son, por una parte, la estructura incipiente de la organización productiva y, por otra, la inestabilidad y escasez de garantías en el empleo. Sin embargo, el mismo organismo internacional, reconoció la evolución del concepto de trabajo informal y desde hace varios años ha utilizado la expresion "sector informal de la economía" para describir las diversas actividades de trabajadores pobres que no son reconocidas, protegidas, ni reguladas por las autoridades públicas, tales como las ventas callejeras, trabajo doméstico, trabajos de empresas explotadoras, trabajos a domicilio, trabajos independientes, microempresas, entre otros.

Asímismo, la OIT distingue dos principales características de los trabajadores informales: la primera se refiere a que este tipo de trabajadores no están reconocidos, ni protegidos dentro de los marcos jurídicos y reglamentarios de los Estados y en segundo lugar, están afectados por un alto nivel de vulnerabilidad, siendo sus labores muy inestables y sus ingresos muy bajos e irregulares (Organización Internacional del Trabajo, 2002)

La OIT acepta que el concepto trabajo informal ha evolucionado y tomado distintas denominaciones, principalmente basadas en las distintas formas de medición del mismo, es así como en 1993, la Conferencia Internacional de Estadísticos del Trabajo (CIET) adoptó una Resolución sobre las estadísticas del empleo llamándolo Sector Informal, por otro lado, en consideración a las características de la unidad de producción, en 2003 se adoptó el concepto de Empleo Informa/ ${ }^{3}$, pero siempre conservando las características del sector y los trabajadores del mismo ya explicada et supra (Oficina Regional para América Latina, 2013, p. 62).

3 De esta manera, el empleo informal fue definido como un conjunto de unidades dedicadas a la producción de bienes 0 la prestación de servicios con la finalidad primordial de generar ingresos para las personas que participan en esa actividad. Estas unidades funcionan típicamente en pequeña escala, con una organización rudimentaria, en la que hay muy poca o ninguna distinción entre el trabajo y el capital como factores de producción. En consecuencia, el empleo informal se refiere a todas aquellas actividades económicas de mercado que operan a partir de los recursos de los hogares, pero sin constituirse como empresas con una personalidad jurídica independiente de esos hogares. 
En Colombia, el Departamento Administrativo Nacional de Estadística (DANE) adopta la definición contenida en la Resolución 15 $5^{\text {a }}$ CIET de la OIT de 1993, la cual menciona que la medición de informalidad en materia de escala de personal ocupado en las empresas debe ser de hasta cinco trabajadores excluyendo los independientes que se dedican a su oficio y a los empleados del gobierno. Por otra parte, se considera que la afiliación al sistema de seguridad social constituye también una aproximación importante al grado de formalidad del empleo (Departamento Administrativo Nacional de Estadística, 2014).

Ahora bien, la definición legal de informalidad laboral en Colombia, ha sido planteada por la Ley 1429 de 2010, como ya se explicó et supra desde dos puntos de vista. El primero, referido a la informalidad con capacidad de acumulación; y el segundo, relacionado a la subsistencia, siendo este último el de interés de esta investigación.

En virtud de lo anterior, se hace necesario revisar los estudios y datos más recientes de los organismos internacionales y locales sobre la situación laboral de los trabajadores informales por subsistencia, pues se constituye en el eje problemático de esta disertación.

En el año 2007, la OIT en coadyuvancia con la Organización Mundial del Comercio (OMC) presentó las conclusiones de un estudio realizado por estos organismos sobre trabajo informal en el mundo y sus consecuencias principales; en torno a dicha investigación se afirma que los trabajadores de la economía informal están desprovistos de seguridad en el empleo, no tienen acceso a prestaciones sociales y sus posibilidades de capacitación y formación son escasas, es decir, no gozan de un trabajo digno. Asimismo, este estudio asevera que un Estado donde exista un alto nivel de economía informal, se limita la proyección y magnitud de las empresas, su productividad, se restringe la capacidad de creación de nuevos puestos de trabajo formales y se estimula la pobreza. Lo anterior, indica que entre mayor economía informal existente en un país, mayor es su índice de pobreza y menor su desarrollo (Organización Internacional del Trabajo y Organización Mundial del Comercio, 2007).

Desde el Banco Mundial (2007) se afirma que en América Latina y el Caribe el empleo informal urbano abarca un $54 \%$, diferenciando dos grupos de ellos, por un lado los trabajadores informales asalariados que representan un $24 \%$, y en segundo lugar los trabajadores informales no asalariados que 
constituyen un $30 \%$ aproximadamente. Asimismo, esta institución indica que este tipo de trabajadores declaran que no contribuyen a los planes de protección social porque sus ingresos son bajos, además de su desconocimiento por los beneficios que ellos traen; y que en países como Colombia las personas dedicadas a las actividades informales registran niveles de satisfacción de necesidades muy bajos (Banco Mundial, 2007).

Refiriéndose al tema, el Banco Interamericano de Desarrollo (BID) en el estudio realizado sobre informalidad laboral (2001) destaca que en casi todos los países de Latinoamérica, con excepción de República Dominicana, hay una mayor participación de mujeres en este tipo de actividades, siendo las más frecuentes, las del servicio doméstico y las actividades por cuenta propia. En el mismo informe se plasma que según Pollak (1993), existe en Latinoamérica un alto número de mujeres desempeñando trabajos informales debido a tres factores: el primero de ellos, se cimienta en el aumento de mano de obra femenina que representa el aumento en la educación y capacitación media por parte de este género, el segundo se refiere a la disminución de los ingresos familiares debido a la crisis económica regional, lo cual exige que la mujer genere recursos para el hogar; y por último, el poco acceso que tienen las mujeres a empleos formales y bien remunerados genera tendencias para desempeñar actividades informales, como posibilidad de empleo (Freije, 2001).

En lo que respecta a Colombia, se debe indicar que según los datos del DANE, la tasa de desempleo ha disminuido en los últimos meses; para el mes de diciembre de 2016 la tasa nacional correspondió al 8,7\%. No obstante, el índice de informalidad laboral ha permanecido muy alto, y en algunas ocasiones aumenta, registrándose actualmente en $48,6 \%$ en el consolidado nacional del cual el $61,1 \%$, corresponde a trabajadores por cuenta propia, enmarcados dentro del concepto de trabajo informal por subsistencia. Asimismo, el informe identifica unos $93,4 \%$ de ocupados afiliados a un régimen de salud y solo el $48 \%$ a un sistema de pensiones (Departamento Administrativo Nacional de Estadística, trimetre móvil octubre-diciembre 2016).

Sobre el tema de informalidad laboral, la Honorable Corte Constitucional colombiana también ha sentado su posición en distintos pronunciamientos. En una de las más recientes Sentencias (T-135 de 2010), reitera su doctrina, indicando que esta es una modalidad de trabajo de la cual dependen muchas familias en Colombia, y que el derecho al trabajo debe ser protegido inclusive cuando riñe con otros derechos fundamentales, tales como los derivados del 
espacio público, cimentando sus afirmaciones en la teoría de la confianza legítima que debe garantizar el Estado a los ciudadanos. También, exhorta a los organismos gubernamentales a que busquen alternativas de reubicación y mejora de las condiciones en que desempeñan sus labores los trabajadores del comercio informal con el ánimo de garantizar sus derechos fundamentales.

Igualmente, el máximo órgano de justicia constitucional ha revalidado la posición del derecho al trabajo como un derecho humano fundamental, caracterizado como un derecho económico y social, que permite que los trabajadores y sus familias vivan en condiciones dignas y justas. Asimismo, ha indicado que el Estado debe formular políticas públicas que efectivicen el derecho del trabajo desde la perspectiva del trabajo decente; esto es, estrategias que reduzcan el desempleo, formalización de puestos de trabajo, garantía de desempeño de actividades laborales en condiciones dignas y justas, entre otras.

Aunado a lo anterior, en sentencia de tutela del año 2013, la Corte Constitucional (C. Const. Sentencia T-386/2013) analizando el tema de la ocupación del espacio público por parte de los vendedores informales ambulantes ha mencionado que el Estado debe ofrecer especial protección a las personas que se dedican a las ventas ambulantes, pues ello obedece principalmente a que se encuentran "en situación de especial vulnerabilidad y debilidad por sus condiciones de pobreza o precariedad económica" y que además, "las personas que se dedican al comercio informal no pueden ser privadas de sus medios de subsistencia, sin que las autoridades les ofrezcan mecanismos adicionales por medio de los cuales puedan satisfacer sus necesidades en forma efectiva y con esto, sus derechos fundamentales como la vida, la dignidad, el mínimo vital, la igualdad, el trabajo, entre otros". De igual manera, la Corte, clasifica a los vendedores informales en razón al lugar y tiempo de ocupación del espacio público así: vendedor estacionario, vendedor informal semiestacionario y vendedor informal ambulante.

Ahora bien, para tener claridad si en Colombia se ha avanzado en el camino hacia la formalización del empleo y el trabajo decente, es pertinente citar de nuevo las conclusiones expuestas por la Procuraduría General de la Nación, mediante su publicación "Trabajo digno y decente en Colombia". En el mencionado informe se asegura que de cada 10 colombianos trabajadores/ as, 6 no tienen un empleo digno; el $63 \%$ de los trabajadores del país ejercen labores de baja calidad, lo que se representa en desigualdad y desprotección laboral. Desde allí, se asevera que el país socialmente es el más 
disímil en América Latina. También, se observa que el $46 \%$ de los asalariados devengan menos de 1 salario mínimo mensual; y que la forma de vinculación por terceros (cooperativas, empresas de servicios temporales, prestación de servicios) ha aumentado a un $27,4 \%$ en 2007 , y la contratación a término fijo ha seguido la misma tendencia en los últimos años (Procuraduría General de la Nación, 2012).

Desde los antecedentes normativos de la Ley 1429 de 2010, y según la exposición de motivos del proyecto de ley 057 de 2010 presentado en la Cámara de Representantes del Congreso y que fue la génesis de la mencionada norma se afirma que "Uno de los principales retos que enfrenta el país actualmente, si no el principal, es la generación de empleo formal," pues se asegura que los antecedentes históricos denotan que una de las mayores dificultades de las empresas que operan en Colombia es la de generar puestos de trabajo formal, especialmente para personas jóvenes (menores de 25 años), cuyas cifras de desempleo son mayores, asimismo se hace prioritario elevar los ingresos y protección de personas y familias más vulnerables y con menor remuneración. Por lo anterior, alcanzar que los trabajadores colombianos, y especialmente los más vulnerables, puedan acceder a empleos formales, con seguridad social y prestaciones que atenúen las contingencias que se presenten en la población, y que cuya remuneración laboral genere estabilidad y bienestar particular y familiar, debe ser el principal objetivo del Estado y de toda la sociedad.

Asimismo, la ponencia del segundo debate en las comisiones terceras del Congreso de la República del proyecto de ley que dio origen a la mencionada ley, afirma que "El Gobierno Nacional a través de su Ministro de la Protección Social, Mauricio Santamaría, presentó el pasado 20 de agosto de 2010 un proyecto de ley que contemplaba cuatro aspectos fundamentales: I) Incentivos para la formalización de empresas micro y pequeñas; ॥) simplificación de trámites para la formalización de las empresas; II) sistema de control para evitar que los beneficios llegaran a empresas fachada y finalmente, IV) Incentivos para impulsar el empleo formal” (Congreso de la República, 2010).

Al respecto, el Consejo de Estado ha mencionado que, con el fin de estimular la formalización empresarial en todos los aspectos y de generar empleo formal, se expidió la Ley 1429 de 2010. Para ello, dicha ley busca generar incentivos en las etapas iniciales de creación de las empresas, "de tal manera que aumenten los beneficios y disminuyan los costos de formalizarse", dado que desde el momento en que se crea una empresa, formalmente existen 
cargas como la matrícula mercantil, el pago de aportes de nómina y el pago de impuestos, que muchos de los pequeños empresarios no pueden asumir, motivo por el cual optan por permanecer en la informalidad, con lo cual causan un grave perjuicio a la economía y al desarrollo del país.

Por su parte la honorable Corte Constitucional en Sentencia C-629 de 2011, afirma que la Ley 1429, se dirige específicamente a facilitar el crecimiento de las pequeñas empresas para que se formalicen y de esta manera generen empleos productivos. Las medidas contempladas se concentran en las microempresas y en las pequeñas empresas porque el $67 \%$ del empleo dependiente o asalariado es generado por este tipo de empresas (según cifras del DANE citadas en la primera ponencia), y porque en este tipo de empresas se concentran los mayores índices de informalidad tanto laboral como empresarial. Así la informalidad empresarial (definida bien sea como la no obtención del registro mercantil o el no pago de impuestos) es del orden del $36 \%$ en las empresas con 10 o menos empleados mientras que ese porcentaje es de alrededor del $7 \%$ para las empresas con más de 10 empleados. Por su parte, la informalidad laboral, definida como el no pago a seguridad social de los trabajadores, es marcadamente mayor en las microempresas (73\%), nuevamente según las cifras del DANE citadas en la ponencia.

Desde las mencionadas explicaciones, se propone el fortalecimiento de los cinco sectores locomotoras de la economía colombiana (Infraestructura, Agro, Vivienda, Minería e Innovación) para que impulsen los sectores altamente generadores de empleo como los servicios y el comercio. Con todo, el proyecto de ley propició los mecanismos que generarían primer empleo y su formalización en Colombia, a través de incentivos económicos para las micro y pequeñas empresas, tales como exención o disminución en el pago de impuestos a la renta, matrícula mercantil, exenciones a las cotizaciones al sistema de seguridad social, disminución de costos de formalización, entre otros, que motivaría a las empresas informales a la formalización, garantizando los derechos laborales de sus trabajadores y, además, generaría nuevos empleos formales (Echeverry Garzón y otros, 2010).

Sin embargo, el impacto de las estrategias implementadas en la Ley 1429 de 2010 según la DIAN y la Unidad de Gestión Pensional y Parafiscales del Ministerio de Hacienda, no han sido efectivas, pues los beneficios de la norma para 239.400 empresas en el primer año de vigencia, solo han sido utilizados para los descuentos tributarios, pero no para formalizar el empleo. Según la entidad, de cada 100 empresas beneficiadas con las prerrogativas norma- 
tivas, solo 3 de ellas hacen los aportes al sistema de seguridad social integral, y el $97,54 \%$ de las mismas tienen 5 trabajadores/as o menos, es decir, no hay generación significativa de empleo (DIAN y UGPP, 2012).

Ahora bien, es imperativo indicar que la mencionada Ley 1429 de 2010 así como las distintas estrategias jurídico-laborales que se han promovido en el país en la última década, como ya se explicó et supra, no han obedecido a un plan nacional de trabajo decente, muy a pesar de que Colombia como estado miembro de la OIT, está obligado a cumplir con el mencionado programa. No obstante, algunas instituciones públicas, académicas y sindicales han propuesto las primeras ideas para la implementación de una política pública nacional de trabajo decente que permita avanzar en garantías laborales mínimas para los trabajadores colombianos, sin tener en cuenta si ejercen un empleo formal o informal.

En tal sentido, la Procuraduría General de la Nación diseñó una propuesta, a partir de la relación estrecha entre tres elementos a saber: El enfoque de derechos, la solidez técnica y la deliberación democrática. La cohesión de estos mecanismos permitirá un pacto social por el trabajo decente, endilgando funciones a cada uno de los actores del contexto laboral; iniciado por el compromiso del Estado a fortalecer las estrategias de generación y formalización del empleo, crear espacios para el diálogo social, la asunción de vigorizar los programas nacionales y locales de emprendimiento, garantizar el acceso masivo a la educación superior, consolidar el sistema de control y vigilancia del Ministerio del Trabajo, brindar capacitación a los funcionarios administrativos y judiciales que vigilan y supervisan el debido respeto a los derechos de los trabajadores/as y asegurar un mínimo de protección a todo aquel que ejerza una actividad de trabajo, sin importar su vinculación al mercado laboral (Procuraduría General de la Nación, 2012).

Desde el ámbito internacional, la OIT ha revisado las estrategias que en los últimos cinco años han sido más significativas y que han tenido mayor impacto en algunos países de América Latina y el Caribe y que les han representado avances significativos en disminución de tasas de desempleo, formalización del empleo, y mejora de condiciones y garantías laborales para los trabajadores. Según el último informe de la OIT para la región (Panorama Laboral, 2013), los países que han adoptado un enfoque global e integrado de sus estrategias para contrarrestar la informalidad han alcanzado mejores resultados, logrando también la creación de empleos formales. El enfoque global se ha desarrollado por siete vías a saber: generación de empleo de calidad 
y estrategias de crecimiento; entorno normativo; diálogo social, organización y representación; fomento de la igualdad y lucha contra la discriminación; medidas de apoyo a la iniciativa empresarial, competencias profesionales y financiación; ampliación de la protección social y estrategias de desarrollo local (Organización Internacional del Trabajo, 2013).

El mismo informe señala que la tasa de trabajo informal en América Latina y el Caribe es de 47,7 \% para el año 2012, identificándose que ha venido decreciendo desde el año 2005 por la aplicación de las estrategias ya mencionadas; sin embargo, se advierte que de no acatarse las recomendaciones de la OIT, podría darse un retroceso en la materia en los próximos años.

No obstante, es importante aclarar que el avance en el asunto no ha sido en común para todos los países latinoamericanos, en los casos de Colombia, Perú, Venezuela, Ecuador, no se han obtenido logros significativos. A pesar, de los múltiples esfuerzos de los Estados y las organizaciones internacionales para mitigar el impacto de la crisis económica mundial de los últimos años sobre el contexto laboral, las formas más precarias de trabajo han aumentado desmesuradamente, destacándose el crecimiento del trabajo informal.

En virtud de los datos anteriores, la OIT lanzó en agosto de 2013 el programa para la promoción de la formalización en América Latina y el Caribe-FORLAC, con la intención de implementar una nueva medida para la reducción de la informalidad laboral y fomento al empleo formal (Organización Internacional del Trabajo, 2014). Al respecto, el informe FORLAC-Colombia expresa que en el país, "el empleo informal no agrícola en Colombia ha disminuido de aproximadamente $58 \%$ en 2009 a $55 \%$ en 2013. A ello, ha contribuido un favorable ciclo económico que estuvo acompañado de generación de empleo, y factores institucionales como la creación del sistema nacional de Mipymes, los acuerdos de formalización laboral y el plan de acción para los derechos laborales, además de programas como "Colombia se formaliza", que han impulsado la formalización empresarial y laboral (Oficina Regional para América Latina y el Caribe, 2014). Empero, se confirma que la informalidad laboral en el país sigue siendo muy alta, y que las distintas estrategias utilizadas por el Estado, no han logrado avances significativos sobre esta problemática y por tanto, aún se está muy lejos de garantizar trabajo decente en el país.

Con todo, se hace necesario develar que solo algunos países latinoamericanos han logrado avances significativos en la reducción de tasas de infor- 
malidad laboral e implementación de planes nacionales de trabajo decente que contribuyan a las garantías adecuadas para la vida del trabajador y su familia. Al respecto, Tokman (2007) expresa que Chile en los últimos 15 años ha registrado la disminución más significativa de la tasa de informalidad en el empleo (32\%), siendo la más baja de la región. Según el autor, estos avances se han basado en aspectos como regulación de la subcontratación, cobertura del sistema de protección social a los informales, y factores asociados al dinamismo de la economía en los últimos años.

\section{CONCLUSIONES}

El avance presentado del proyecto de tesis doctoral, colige en sus primeros resultados que en Colombia se hace necesario e inmediato, diseñar y proponer una estrategia jurídico-laboral de trabajo decente que permita al Estado implementar mecanismos eficaces para la garantía de condiciones laborales mínimas de los/las trabajadores/as que desempeñan actividades informales enmarcadas dentro del concepto legal de informalidad por subsistencia, teniendo en cuenta que las medidas implementadas por la Ley 1429 de 2010, no han sido suficientes para reducir las altas tasas de informalidad laboral en el país, además, porque no han sido planificadas desde un programa nacional orientado a desarrollar el programa de trabajo decente propuesto por la OIT.

No se desconocen los esfuerzos de formalización laboral que ha implementado el Ministerio del Trabajo en Colombia, para diversos sectores de trabajo, así como la ratificación de normas internacionales y la expedición de normas locales orientadas con el mismo objetivo, sin embargo, como se argumenta durante el texto, los trabajos informales en Colombia se diversifican en sus modalidades, y continúan siendo la única salida de subsistencia para la mitad de las personas ocupadas. Se espera que la respuesta a la pregunta de investigación planteada pueda ser respondida integralmente con la finalización del trabajo de tesis.

Con todo, se puede concluir que son pocos los estudios nacionales que enlazan la problemática de la informalidad laboral como mecanismo para generar ingresos que satisfagan las necesidades básicas del individuo y su familia, pues aunque se advierten algunas investigaciones regionales principalmente desde la academia, y algunos datos estadísticos nacionales suministrados por el DANE, no se evidencia un diagnóstico actual y específico de la situación, ni tampoco estrategias jurídico-laborales encaminadas a promover eficazmente el trabajo decente de los trabajadores informales por subsistencia. 


\section{REFERENCIAS BIBLIOGRÁFICAS}

Banco Mundial (2007). Informalidad: escape y exclusión. Washington: Banco Mundial.

Borrego, N. S. (2006). Mercado de trabajo e informalidad: Repercusiones en la seguridad social. Gaceta Laboral.

Cárcamo, H. (2005). Hermenéutica y análisis cualitativo. Cinta de Moebio, 14.

Cervantes, Gutiérrez y Palacios J. (2008). El concepto de la economía informal y su aplicación en México. Estudios demográficos y urbanos, 21-54.

Colombia. Corte Constitucional. Sentencia C-606 de 1992. M.P. Ciro Angarita Barón. La jurisprudencia de la Corte Constitucional frente a la creación del trabajo formal. Bogotá. Recuperado el 10 de 10 de 2014, de http:// www.corteconstitucional.gov.co/viencuentro/conferencia\%20gemm. php

Colombia. Corte Constitucional. Sentencia C-629 de 29 de julio de 2003. M.P. Álvaro Tafur Galvis.

Colombia. Corte Constitucional. Sentencia T-135 de 24 de octubre de 2010. M.P. Gabriel Eduardo Mendoza Martelo. Recuperado de: http://www. corteconstitucional.gov.co/relatoria/2010/T-135-10.htm

Colombia. Corte Constitucional. Sentencia T-152 de 07 de marzo de 2011. M.P. Alejandro Linares Cantillo. Recuperado de: https://corte-constitucional.vlex.com.co/vid/680009365

Colombia. Corte Constitucional. Sentencia T-386 de 28 de junio de 2013. M.P. María Victoria Calle Correa. Recuperado de. https://corte-constitucional.vlex.com.co/vid/-474627142

Colombia. Decreto 2025 de 2011 (sobre contratación a través de cooperativas y precooperativas de trabajo asociado). Bogotá: Editorial Legis.

Colombia. Ley 1429 de 2010. Bogotá: Editorial Leyer.

Colombia. Ley 1438 de 2011 (reforma al sistema de salud). Bogotá: Editorial Leyer.

Colombia. Ley 1468 de 2011. Bogotá: Editorial Leyer.

Colombia. Ley 1496 de 2011 (sobre equidad salarial para la mujer). Bogotá: Editorial Legis.

Colombia. Ley 1788 de 2016. Bogotá: Editorial Legis.

Colombia. Ley 1822 de 2017 (sobre licencia de maternidad). Bogotá: Editorial Leyer.

Comisión Europea, Dirección General de Empleo, Relaciones Laborales y Asuntos Sociales (1998). Glosario de términos relativos a la igualdad. Glosario de términos relativos a la igualdad entre hombres y mujeres. Recuperado el 01 de 03 de 2012, de: http://www.europarl.europa.eu/ transl_es/plataforma/pagina/celter/glosario_genero.htm\#G

Consejo de Estado. Beneficio de progresividad en el pago del impuesto sobre la renta y complementarios de la Ley 1429 de 2010, 11001-0327-000-2012-00006-00(19306) 23 de mayo de 2013. 
Charmes, J. (2000). Women in Informal Employment: Globalizing and Organizing (WIEGO). Segunda reunión anual. Cambridge, Massachusetts.

DANE. (junio de 2013). Departamento Administrativo Nacional de Estadística. DANE. Recuperado el 10 de junio de 2014, de DANE: file://C:/Users/ Estudiante/Downloads/bol_regiones_Isem_13.pdf

Departamento Administrativo Nacional de Estadística (2012). Medición del empleo informal y seguridad social: Trimestre móvil-mayo-julio 2012. Bogotá: DANE.

Departamento Administrativo Nacional de Estadística (2012). Mercado laboral por sexo: Trimestre móvil mayo-julio 2012. Bogotá: DANE.

Departamento Administrativo Nacional de Estadística (2014). Informe trimestre móvil mayo-julio 2014. Bogotá: DANE.

Departamento Administrativo Nacional de Estadística (2016). Medición del empleo, informalidad laboral y seguridad social: Trimestre móvil octubre-diciembre 2016. Bogotá: DANE.

Departamento Administrativo Nacional de Estadística (2017). Medición del empleo informal y seguridad social. Bogotá: DANE.

DIAN y UGPP (30 de 03 de 2012). Comunicado de prensa. Obtenido de http://www.ugpp. gov.co/: http://www.ugpp. gov.co/doc_view/429 -comunicado-de-prensa-dian-ugpp

Echeverry Garzón, J. C. y otros (2010). Proyecto de ley 057. Bogotá: Temis.

Escuela Nacional Sindical (2011). Informe nacional de trabajo decente 2010. Medellín: ESN.

Espinoza, M. (2003). Trabajo decente y protección social. Recuperado el 23 de 03 de 2012, de: OIT Chile: http://www.oitchile.cl/pdf/publicaciones/ ser/ser007.pdf

Federación Internacional de Sociedades de la Cruz Roja y de la Media Luna Roja (s.f.). Recuperado el 7 de mayo de 2014, de: Gestión de desastres población desplazada: http://www.ifrc.org/es/introduccion/disaster-management/sobre-desastres/definicion--de-peligro/ desplazamiento-de-poblaciones-y-personas-desplazadas/

Fedesarrollo (2013). Reforma tributaria y mercado laboral. Bogotá: Editorial Temis.

Freije, S. (2001). Banco Interamericano de desarrollo. El empleo informal en América Latina y el Caribe: Causas, consecuencias y recomendaciones. Washington: BID.

Fundación Corona (junio de 2012). Recuperado el 07 de mayo de 2014, de Evaluación a la Calidad de Vida en Ibagué 2008-2011: http://www. fundacioncorona.org.co/templates/publicaciones. php?search=Evaluaci $\%$ F3n $\% 20$ de $\% 20 l a \% 20$ calidad $\% 20$ de $\% 20$ vida $\% 20$ en $\% 20$ lbagu\%E9\%20\%202008\%20-\%202011

Galvis, L. (2012). Informalidad laboral en áreas urbanas de Colombia. CEER. Bogotá: Editorial Temis.

Ghai, D. (2003). La medición del trabajo decente. Revista Internacional del Trabajo, 122(2), 122-156. 
Guataquí, J. (2010). El perfil de la informalidad en Colombia. Universidad de Antioquia.

Hernández, R., Fernández, C. \& Baptista, P. (2003). Metodología de la investigación. México: McGraw-Hill.

Ministerio de Relaciones Exteriores y Ministerio de la Protección Social (06 de 2012). La situación de Colombia frente a la comisión de aplicación de normas de la Conferencia Internacional de Trabajo. Obtenido de http:// www.derechoshumanos.gov.co/Prensa/Destacados/Documents/2010/ destacados/OIT-Comisi\%C3\%B3n\%20de\%20Normas-Rev\%20AMH-10 $\% 2006 \% 2010$.pdf

Mintrabajo (junio de 2013). Ministerio de Trabajo. Recuperado el 11 de junio de 2014, de file:///C:/Users/Estudiante/Downloads/ PlanEmpleoTolima_2013.pdf

Organización de Naciones Unidas (16 de 12 de 1966). Pacto Internacional de Derechos Económicos, Sociales y Culturales.

Oficina Internacional del Trabajo (2002). El trabajo decente y la economía informal. Conferencia 90. Ginebra, Suiza.

Oficina Internacional del Trabajo (2013). Economía informal y trabajo decente. Transición hacia la formalidad. Ginebra: International Labour Office.

Organización Internacional del Trabajo (2013). La OIT en América Latina y el Caribe, avances y perspectivas. OIT.

Organización Internacional del Trabajo (s.f.). Recuperado el 07 de mayo de 2014, de Trabajo Decente: http://ilo.org/global/topics/decent-work/ lang--es/index.htm

Organización Internacional del Trabajo (1991). El dilema del sector no estructurado. Conferencia 78. Ginebra: OIT.

Organización Internacional del Trabajo (20 de 09 de 2014). Obtenido de http://www.ilo.org/dyn/normlex/es/f?p=NORMLEXPUB:11200:0::NO:: P11200_COUNTRY_ID:102595

Organización Internacional del Trabajo (20 de 09 de 2014). Organización Internacional del Trabajo. Recuperado de: http://ilo.org/americas/ temas/econom\%C3\%ADa-informal/lang--es/index.htm

Organización Internacional del Trabajo (2013). Panorama Laboral 2013-América Latina y el Caribe. Perú: OIT. Recuperado de. http:// studylib.es/doc/6881333/panorama-laboral-2013---am\%C3\%A9rica -latina-y-el-caribe--pdf

Organización Internacional del Trabajo y Organización Mundial del Comercio (2007). La globalización y el empleo en el sector informal en los países en desarrollo. Ginebra: OIT y OMC.

Oficina Regional para América Latina y el Caribe (2013). Panorama Laboral 2013-América Latina y el Caribe. Lima: Organización Internacional del Trabajo.

Oficina Regional para América Latina y el Caribe (2014). Evolución del empleo informal en Colombia: 2009-2013. Lima-Perú: OIT.

Oficina Regional para América Latina y el Caribe (2014). Notas sobre formalización laboral. Perú: Organización Internacional del Trabajo. 
Pippidi, A. (2000). In the shadows: Informal economy and the survival strategies. Rumania.

Portes, A., Castells, M. \& Benton, L. (1989). The Informal Economy: Studies in Advanced and Less Developed Countries. Londres: The Johns Hopkins University Press.

Procuraduría General de la Nación (2012). Trabajo digno y decente en Colombia: seguimiento y control preventivo a las políticas públicas. Bogotá: Procuraduría General.

Rodríguez, I. (27 de noviembre de 2012). JA TELINE. Recuperado el 11 de junio de 2014, de Periodicoelector: http://periodicoelector.com/ index.php/de-interes/economia/1805-cundinamarca-subcampeon-de -la-informalidad-laboral

Somavia, J. (1999). Conferencia internacional del trabajo. Ginebra: OIT.

Tokman, V. (2007). Informalidad, inseguridad y cohesión social en América Latina. Santiago de Chile: CEPAL.

Uribe, J. \& otros (09 de 2006). Una teoría general sobre la informalidad: el caso colombiano. En: Economía y Desarrollo, 5(2), 213-273.

Villavicencio, A. \& otros (2010). Trabajo decente: Subregional, Bolivia, Colombia, Ecuador y Perú. Programa laboral para el desarrollo. Perú: Plades. 